\title{
Extracranial Metastases of a Cerebral Glioblastoma: A Case Report and Review of the Literature
}

\author{
Jurij Rosen ${ }^{a}$ Tobias Blau $^{b}$ Stefan J. Grau ${ }^{c} \quad$ Michael T. Barbe $^{a}$ \\ Gereon R. Fink ${ }^{a}$ d Norbert Galldiks ${ }^{a}$, e \\ aDepartment of Neurology, University of Cologne, Cologne, Germany; binstitute of \\ Neuropathology, University of Cologne, Cologne, Germany; 'Department of \\ Neurosurgery, University of Cologne, Cologne, Germany; IInstitute of Neuroscience and \\ Medicine (INM-3), Forschungszentrum Jülich, Jülich, Germany; ${ }^{e}$ Center of Integrated \\ Oncology (ClO), University of Cologne, Cologne, Germany
}

\section{Keywords}

Glioma · Extraneural metastasis · Radiotherapy · Chemotherapy · Tumor resection

\begin{abstract}
The glioblastoma, a malignant human brain tumor, is known for its devastating intracranial progress and its dismal prognosis. Whereas treatment and research are most prominently focused on the primary tumor lesion, in recent years evidence has accumulated that points to the rare occurrence of extracranial glioblastoma metastases. We here present a case of a female patient with a known glioblastoma who was detected to harbor multiple metastases in the bones, lung, pleura, liver, mesentery, and the subcutaneous soft tissue. Pathogenetically, these metastatic lesions developed most probably after a local progression of the left temporal glioblastoma through the skull base, thus getting access to the systemic lymphatics. Similar cases of extensive glioblastoma metastization, their putative underlying mechanisms, and implications for clinical care are discussed.




\section{Case Reports in Oncology}

\section{Introduction}

The glioblastoma (GBM) is the most common and most malignant brain tumor in adults with an unfavorable prognosis despite multimodal treatment. Relatively little is known about the occurrence of extracranial GBM metastases and the underlying pathogenesis. Whereas in 1928 Davis [1] was the first to present a case of a metastasized GBM, a growing body of case reports has more recently pointed to a putatively higher incidence of systemic metastases in GBM patients than hitherto assumed [2-7].

Nevertheless, the overall number of only approximately 200 published cases underlines both the rarity of extracranial GBM metastases and the to date unclear pathogenesis $[6,8,9]$. Epidemiologically, younger and otherwise healthier patients were shown to be susceptible to develop extracranial metastases, most probably due to a longer overall survival (OS) compared to elderly GBM patients with multiple chronic illnesses. The most parsimonious explanation for this observation is that younger patients offer more time for tumor cells to escape from the brain and to form distant metastases $[6,7]$. However, the fact that even in young GBM patients the occurrence of extracranial metastases is rare strongly suggests that other factors than time from onset of the disease are relevant for the relatively rare incidence of extracranial GBM metastization, also in relation to other tumor entities. It is broadly assumed that physical hurdles inherent to the cerebral environment, e.g., the dura mater, the thickened basement membrane, and the blood-brain barrier, all help to prevent tumor cells from spreading beyond the brain $[4,6,10,11]$. Moreover, the missing connection between intra- and extracranial perivascular spaces as well as an anatomically improbable invasion of thin-walled intracerebral veins by the tumor may render metastasogenesis difficult [12]. However, these factors cannot solely account for the rare metastasogenesis, as roughly $20 \%$ of GBM patients were shown to harbor circulating tumor cells (CTC) in the peripheral blood, an incidence comparable to CTC counts detected in patients with other carcinoma without metastases [5, 1315]. Consequently, CTC of the GBM seem to be hindered to get access to other organs, putatively explained by intrinsic properties of the glial filaments, a lack of specific extracellular matrix proteins, or a multistage peripheral immune response [10,11,15-17]. Lastly, as already mentioned above, extraneural metastases of the GBM may be rare due to the rapid intracranial progression of the primary tumor leaving peripherally CTC not sufficient time for successful systemic metastasogenesis $[6,11]$.

Various mechanisms have been hypothesized to underlie extracranial GBM metastasogenesis. In particular, hematogenous spread via tumorous neovascularization is thought to constitute one main route for metastasogenesis $[4,6,14,18]$. In this context, radiotherapy is assumed to promote vascular invasion and tumor dissemination by both causing metaplasia of the tumor cells and breaching natural barriers $[11,19]$. Besides irradiation, tumor surgery has intensively been discussed as another putative factor facilitating tumor spread since the ensuing disruption of the dura might leave behind an escape route for remaining tumor cells [20]. Yet, no significant differences of CTC counts have been detected in patients before, during, or after tumor resection, indicating that a critical role of surgery facilitating metastasogenesis to date remains to be proven [15]. Furthermore, systemic dissemination of tumor cells by the lymphatic system may also be considered to constitute a possible route for tumor spread given the accumulating evidence pointing to the existence of intradural lymphatic vessels in the brain [21]. Rather theoretically, given the micro- and macroanatomical barriers 
of the brain, local tumor progression was hypothesized to cause systemic metastases [14, $22,23]$.

Once the barriers preventing metastization have been overcome successfully, GBM tumor cells are most prominently found in the lymph nodes, bone, lung, liver, soft tissue, and the skin. However, a specific order of the most frequently metastasized organs has not yet been established $[6-9,13,24]$.

To the best of our knowledge, data on multiple organ metastization by extraneural GBM are scarce. We here report on a GBM patient with multivisceral extraneural metastases and review the literature regarding multiple organ manifestation by GBM tumor cells.

\section{Case Presentation}

A 48-year-old female patient with no significant past medical history presented with progressive cognitive deficits, deterioration of speech, and a new right-sided hemiparesis. Furthermore, a newly diagnosed right-sided hemiparesis led to a cerebral MRI and revealed a left temporal lesion suspicious of a GBM (Fig. 1a). After partial resection, the symptoms improved, and neuropathological examination confirmed a GBM (MGMT promoter not methylated, IDH$1 /-2$ wild type). Afterwards, radiotherapy (total dose, $60 \mathrm{~Gy}$ ) with concomitant temozolomide chemotherapy (daily dose of $150 \mathrm{mg}$ over 6 weeks) was initiated. Due to unwanted and intolerable side effects (i.e., loss of appetite, nausea, vomiting), adjuvant temozolomide chemotherapy was paused after the first cycle.

Two months later, the patient presented with recurrent disturbance of speech and memory deficits. Follow-up MRI revealed local tumor progression, and a second resection was performed. Neuropathological findings were consistent with a GBM relapse. Consequently, adjuvant chemotherapy with temozolomide was re-initiated, which was now tolerated and followed by an improvement of speech and cognition. Five months after the initial diagnosis of the GBM, the patient complained of a persistent local pain in the left maxilla. The assumption of an underlying dental pathology led to a dental extraction, which, however, did not remove the pain. Subsequent MR imaging rather showed a tumor progression through the skull base into the pterygopalatine fossa with infiltration of the soft tissue (Fig. 1b). Additionally, a lymph node metastasis of the neck was detected, most probably due to a GBM tumor cell infiltration of local lymphatic vessels (Fig. 1c).

Consequently, a second palliative radiotherapy with a cumulative dose of 52 Gy of the neck region followed by adjuvant chemotherapy with lomustine was initiated. Despite these efforts, the patient suffered from progressive speech disturbances and nausea, which could be explained by an extensive progression of the tumor lesion inside and outside the cranium (Fig. 1d). Antiangiogenic therapy with bevacizumab was initiated.

Only 3 weeks after the initiation of bevacizumab treatment, the patient suffered from a severe intestinal hemorrhage. Despite a CT scan of the thorax and abdomen, colono-, and gastroscopy, no source of the bleeding could be detected. Fortunately, the bleeding ceased spontaneously.

Unexpectedly, however, the CT scan detected multiple lesions in the lung, pleura, liver, mesentery as well as osteolyses of the pelvis, the seventh rib on the left, and the second lumbar vertebral body, all suspicious of being metastases (Fig. 2). Moreover, a suspect subcutaneous 


\section{Case Reports in Oncology}

lesion located at the right upper arm was palpable. The pathological examination of another subcutaneous lesion located below the left costal arch demonstrated the same histological characteristics as the intracranial GBM (Fig. 3). Subsequently, the patient was admitted to palliative care and died due to intracranial progression of the tumor 13 months after initial diagnosis.

\section{Discussion}

We present a rare case of an extensive metastasization of an intracranial GBM. In contrast to the majority of so far published similar cases (Table 1), the present report suggests a comprehensive and well-documented route of systemic metastasogenesis. After a per continuitatem progression of the GBM through the skull base, its cells invaded cervical lymphatic vessels, followed by a systemic metastatic spread. Given the histological characteristics of the intracerebral GBM, the identical histological findings of one biopsied, extracranial metastasis, and the clinical course, the present case meets (except autopsy) the criteria defined by Weiss in 1954 for a metastasized cerebral glioma [25].

Given our current knowledge of extraneural GBM metastasogenesis and of the clinical course of affected patients, the question arises whether specific therapeutic procedures can be inferred that may help to prevent metastatic spread. As mentioned above, surgical resection of the primary tumor has intensively been discussed as an element facilitating systemic metastasization. However, although earlier case reports suggested that craniotomy may constitute one factor triggering extracranial tumor spread, more recent reports question a causal role of craniotomy in extracranial metastization $[12,14,15,26]$. It seems plausible to assume that rather than the surgical manipulation per se a more aggressive tumor cell type may lead to a rapid and extensive growth of the intracranial lesion necessitating further surgical interventions, which may be responsible for the coincidence of surgery and metastasization [23, 27]. Conversely, as patients undergoing surgery were shown to have a longer OS compared to patients without surgical intervention, the prolonged OS could eventually provide more time for metastization, hence resulting in a coincidence of neurosurgical tumor resection and GBM metastases [14]. Taken together, at present it seems most plausible that neurosurgery might coincide with rather than trigger metastasogenesis.

As another therapeutic approach, in single cases chemotherapy was shown to be the only therapy option that might prolong the time to diagnosis of metastasis and improve the clinical condition $[6,26]$. In stark contrast, a putative facilitating effect for metastasogenesis has been suggested for certain chemotherapeutic agents $[11,19,28]$. However, a structured treatment regimen including temozolomide chemotherapy is standard in the care of GBM patients [29].

So far, extracranial GBM metastization is thought to play a minor role for the clinical course and prognosis of affected patients. On the other hand, this phenomenon is putatively more common than assumed as systemic metastases of the GBM are found in $6-25 \%$ of autopsies of affected patients [15, 22, 30,31]. Thus, in the future a potential increase in OS of GBM patients due to novel treatment options might provide more time for extracranial metastization. As a consequence, extracranial metastatic lesions putatively may occur more and gain clinical importance $[4,14,26,27,30]$. Furthermore, improved multimodal diagnostics might 
also increase the number of detected cases resulting in an improved awareness of the existence of extracranial metastases $[4,6,9,18]$.

As a consequence, besides standard treatment or the inclusion in studies and early palliative care in GBM patients, the clinician's attention also needs to be drawn towards the potential existence of extracranial GBM metastases, thereby allowing earlier diagnosis and thus appropriate treatment adjustment, resulting in improved overall quality of life. For instance, GBM patients with newly diagnosed bone or back pain, dyspnea, or abnormalities in laboratory tests (e.g., anemia, thrombocytopenia, hypercalcemia, etc.) should sooner be admitted to appropriate diagnostics $[18,32]$. In the present case, for instance, the painful dental extraction due to misdiagnosis of the local pain at the left maxilla could have been omitted and a more appropriate treatment could have been initiated without further loss of time.

In summary, extracranial GBM metastases seem to occur more often than widely assumed. To date, the pathogenesis of such metastases remains elusive. Our case shows that an increased awareness of extracranial GBM metastases may help to prevent misdiagnosis and ensuing unneeded procedures and may thereby lead to earlier correct diagnosis and treatment as well as improved quality of life.

\section{Statement of Ethics}

The patient's husband (and authorized representative) gave written informed consent for participation in the study and publication of the present case report.

\section{Disclosure Statement}

Related to the present work, the authors disclose no potential conflicts of interest. The authors declare that they have no competing interests.

\section{Funding Sources}

No funding was received.

\section{Author Contributions}

J.R. and N.G. wrote the manuscript, T.B. analyzed and contributed the histological sections, G.R.F., S.J.G., and M.T.B. scrutinized and revised the manuscript. All authors read and approved the final manuscript. 


\section{References}

1 Davis L. Spongioblastoma Multiforme of the Brain. Ann Surg. 1928 Jan;87(1):8-14.

2 Saad AG, Sachs J, Turner CD, Proctor M, Marcus KJ, Wang L, et al. Extracranial metastases of glioblastoma in a child: case report and review of the literature. J Pediatr Hematol Oncol. 2007 Mar;29(3):190-4.

3 Mentrikoski M, Johnson MD, Korones DN, Scott GA. Glioblastoma multiforme in skin: a report of 2 cases and review of the literature. Am J Dermatopathol. 2008 Aug;30(4):381-4.

4 Piccirilli M, Brunetto GM, Rocchi G, Giangaspero F, Salvati M. Extra central nervous system metastases from cerebral glioblastoma multiforme in elderly patients. Clinico-pathological remarks on our series of seven cases and critical review of the literature. Tumori. 2008 Jan-Feb;94(1):40-51.

5 Lun M, Lok E, Gautam S, Wu E, Wong ET. The natural history of extracranial metastasis from glioblastoma multiforme. J Neurooncol. 2011 Nov;105(2):261-73.

6 Kalokhe G, Grimm SA, Chandler JP, Helenowski I, Rademaker A, Raizer JJ. Metastatic glioblastoma: case presentations and a review of the literature. J Neurooncol. 2012 Mar;107(1):21-7.

7 Blume C, von Lehe M, van Landeghem F, Greschus S, Boström J. Extracranial glioblastoma with synchronous metastases in the lung, pulmonary lymph nodes, vertebrae, cervical muscles and epidural space in a young patient - case report and review of literature. BMC Res Notes. 2013 Jul;6(1):290.

8 Kup PG, Nieder C, Winnekendonk G, Adamietz IA, Fakhrian K. Extracranial oral cavity metastasis from glioblastoma multiforme: A case report. Mol Clin Oncol. 2016 Oct;5(4):437-9.

9 Pietschmann S, von Bueren AO, Kerber MJ, Baumert BG, Kortmann RD, Müller K. An individual patient data meta-analysis on characteristics, treatments and outcomes of glioblastoma/ gliosarcoma patients with metastases outside of the central nervous system. PLoS One. 2015 Apr;10(4):e0121592.

10 Mourad PD, Farrell L, Stamps LD, Chicoine MR, Silbergeld DL. Why are systemic glioblastoma metastases rare? Systemic and cerebral growth of mouse glioblastoma. Surg Neurol. 2005 Jun;63(6):511-9.

11 Ray A, Manjila S, Hdeib AM, Radhakrishnan A, Nock CJ, Cohen ML, et al. Extracranial metastasis of gliobastoma: three illustrative cases and current review of the molecular pathology and management strategies. Mol Clin Oncol. 2015 May;3(3):479-86.

12 Smith DR, Hardman JM, Earle KM. Metastasizing neuroectodermal tumors of the central nervous system. J Neurosurg. 1969 Jul;31(1):50-8.

13 Anghileri E, Castiglione M, Nunziata R, Boffano C, Nazzi V, Acerbi F, et al. Extraneural metastases in glioblastoma patients: two cases with YKL-40-positive glioblastomas and a meta-analysis of the literature. Neurosurg Rev. 2016 Jan;39(1):37-45; discussion 45-6.

14 Liwnicz BH, Rubinstein LJ. The pathways of extraneural spread in metastasizing gliomas: a report of three cases and critical review of the literature. Hum Pathol. 1979 Jul;10(4):453-67.

15 Müller C, Holtschmidt J, Auer M, Heitzer E, Lamszus K, Schulte A, et al. Hematogenous dissemination of glioblastoma multiforme. Sci Transl Med. 2014 Jul;6(247):247ra101.

16 Hulbanni S, Goodman PA. Glioblastoma multiforme with extraneural metastases in the absence of previous surgery. Cancer. 1976 Mar;37(3):1577-83.

17 Wakamatsu T, Matsuo T, Kawano S, Teramoto S, Matsumura H. Glioblastoma with extracranial metastasis through ventriculopleural shunt. Case report. J Neurosurg. 1971 May;34(5):697-701.

18 Goodwin CR, Liang L, Abu-Bonsrah N, Hdeib A, Elder BD, Kosztowski T, et al. Extraneural Glioblastoma Multiforme Vertebral Metastasis. World Neurosurg. 2016 May;89:578-82.e3.

19 Horiuchi T, Osawa M, Itoh N, Kobayashi S, Nitta J, Hongo K. Extradural extension of glioblastoma multiforme into the oral cavity: case report. Surg Neurol. 1996 Jul;46(1):42-6.

20 Beauchesne P. Extra-neural metastases of malignant gliomas: myth or reality? Cancers (Basel). 2011 Jan;3(1):461-77.

21 Sun BL, Wang LH, Yang T, Sun JY, Mao LL, Yang MF, et al. Lymphatic drainage system of the brain: A novel target for intervention of neurological diseases. Prog Neurobiol. 2018 Apr-May;163-4:118-43

22 Pompili A, Calvosa F, Caroli F, Mastrostefano R, Occhipinti E, Raus L, et al. The transdural extension of gliomas. J Neurooncol. 1993 Jan;15(1):67-74.

23 Sanerkin NG. Transdural spread of glioblastoma multiforme. J Pathol Bacteriol. 1962 Jul;84(1):228-33.

24 Pasquier B, Pasquier D, N'Golet A, Panh MH, Couderc P. Extraneural metastases of astrocytomas and glioblastomas: clinicopathological study of two cases and review of literature. Cancer. 1980 Jan;45(1): 112-25.

25 Weiss L. A metastasizing ependymoma of the cauda equina. Cancer. 1955 Jan-Feb;8(1):161-71.

26 Astner ST, Pihusch R, Nieder C, Rachinger W, Lohner H, Tonn JC, et al. Extensive local and systemic therapy in extraneural metastasized glioblastoma multiforme. Anticancer Res. 2006 Nov-Dec;26 6C:4917-20. 
27 Alatakis S, Malham GM, Thien C. Spinal leptomeningeal metastasis from cerebral glioblastoma multiforme presenting with radicular pain: case report and literature review. Surg Neurol. 2001 Jul;56(1):33-7.

28 Terheggen HG, Müller W. Extracerebrospinal metastases in glioblastoma. Case report and review of the literature. Eur J Pediatr. 1977 Jan;124(2):155-64.

29 Stupp R, Mason WP, van den Bent MJ, Weller M, Fisher B, Taphoorn MJ, et al.; European Organisation for Research and Treatment of Cancer Brain Tumor and Radiotherapy Groups; National Cancer Institute of Canada Clinical Trials Group. Radiotherapy plus concomitant and adjuvant temozolomide for glioblastoma. N Engl J Med. 2005 Mar;352(10):987-96.

30 Lombard A, Goffart N, Rogister B. Glioblastoma Circulating Cells: Reality, Trap or Illusion? Stem Cells Int. 2015;2015:182985.

31 Vertosick FT Jr, Selker RG: Brain stem and spinal metastases of supratentorial glioblastoma multiforme: a clinical series. Neurosurgery. 1990 0ct;27(4):516-21; discussion 521-2.

32 Cooper PR, Budzilovich GN, Berczeller PH, Lieberman A, Battista A. Metastatic glioma associated with hypercalcemia. Report of two cases. J Neurosurg. 1974 Feb;40(2):255-9.

33 Wisiol ES, Handler S, French LA. Extracranial metastases of a glioblastoma multiforme. J Neurosurg. 1962 Mar;19(3):186-94.

34 Makeever LC, King JD. Medulloblastoma with extracranial metastasis through a ventriculovenous shunt. Report of a case and review of the literature. Am J Clin Pathol. 1966 Aug;46(2):245-9.

35 Rubinstein LJ. Development of extracranial metastases from a malignant astrocytoma in the absence of previous craniotomy. Case report. J Neurosurg. 1967 May;26(5):542-7.

36 Yokoyama H, Ono H, Mori K, Kishikawa M, Kihara M. Extracranial metastasis of glioblastoma with sarcomatous component. Surg Neurol. 1985 Dec;24(6):641-5.

37 Wallace CJ, Forsyth PA, Edwards DR. Lymph node metastases from glioblastoma multiforme. AJNR Am J Neuroradiol. 1996 Nov-Dec;17(10):1929-31.

38 Beauchesne P, Soler C, Mosnier JF. Diffuse vertebral body metastasis from a glioblastoma multiforme: a technetium-99m Sestamibi single-photon emission computerized tomography study. J Neurosurg. 2000 Nov;93(5):887-90.

39 Houston SC, Crocker IR, Brat DJ, Olson JJ. Extraneural metastatic glioblastoma after interstitial brachytherapy. Int J Radiat Oncol Biol Phys. 2000 Oct;48(3):831-6.

40 Ueda S, Mineta T, Suzuyama K, Furuta M, Shiraishi T, Tabuchi K. Biologic characterization of a secondary glioblastoma with extracranial progression and systemic metastasis. Neuro Oncol. 2003 Jan;5(1):14-8.

41 Mirzayan MJ, Samii M, Petrich T, Börner AR, Knapp WH, Samii A. Detection of multiple extracranial metastases from glioblastoma multiforme by means of whole-body [18F]FDG-PET. Eur J Nucl Med Mol Imaging. 2005 Jul;32(7):853.

42 Didelot A, Taillandier L, Grignon Y, Vespignani H, Beauchesne P. Concomitant bone marrow metastasis of a glioblastoma multiforme revealed at the diagnosis. Acta Neurochir (Wien). 2006 Sep;148(9):997-1000.

43 Mujic A, Hunn A, Taylor AB, Lowenthal RM. Extracranial metastases of a glioblastoma multiforme to the pleura, small bowel and pancreas. J Clin Neurosci. 2006 Jul;13(6):677-81.

44 Hamilton JD, Rapp M, Schneiderhan T, Sabel M, Hayman A, Scherer A, et al. Glioblastoma multiforme metastasis outside the CNS: three case reports and possible mechanisms of escape. J Clin Oncol. 2014 Aug;32(22):e80-4. 


\section{Case Reports in Oncology}

Case Rep Oncol 2018;11:591-600

DOI: $10.1159 / 00049211$

(c) 2018 The Author(s). Published by S. Karger AG, Basel www.karger.com/cro

Rosen et al.: Extracranial Metastases of a Cerebral Glioblastoma
At initial diagnosis

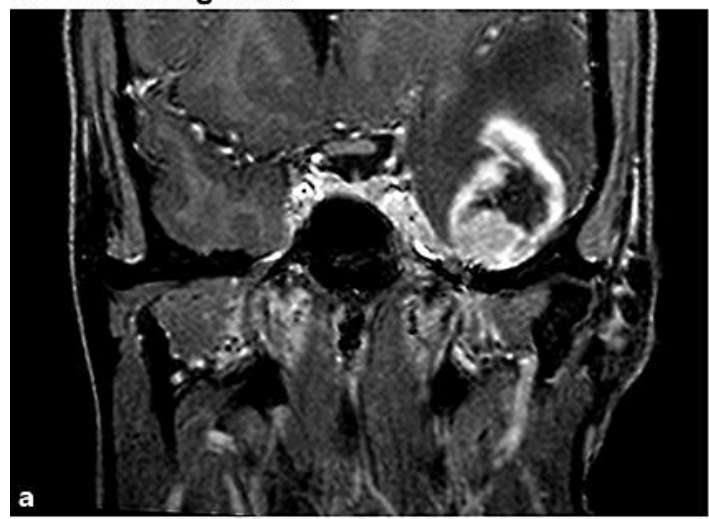

6 months later

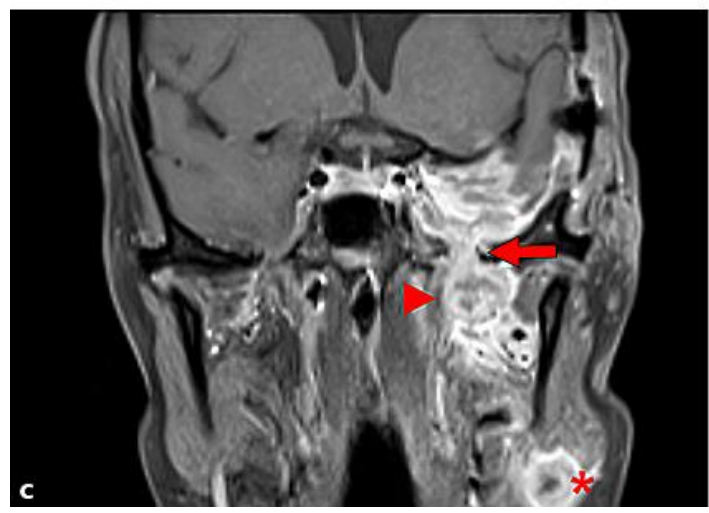

5 months later

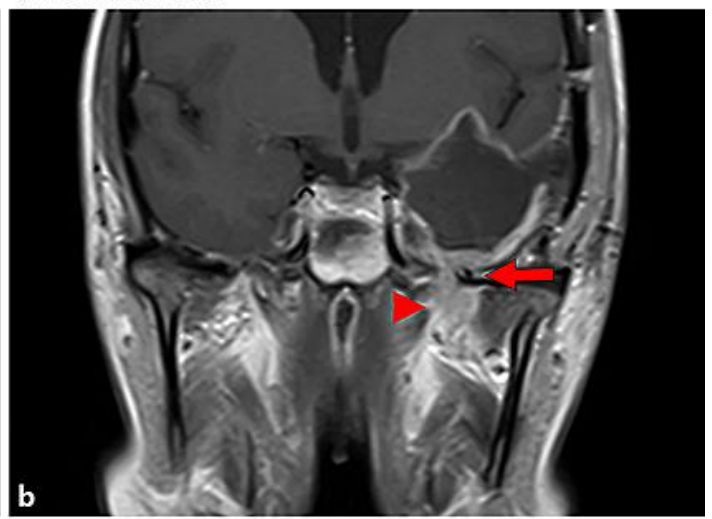

10 months later

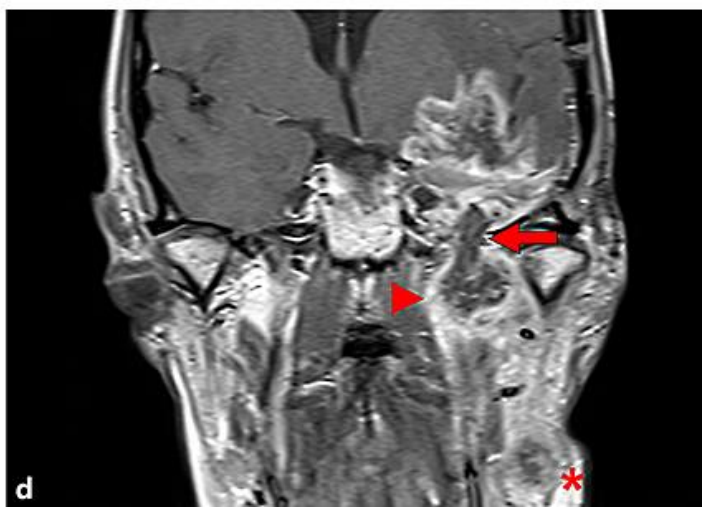

Fig. 1. Coronal sections of serial brain MRI scans showing the local progression of the GBM. a At the initial diagnosis. $\mathbf{b}$ Following resection of the tumor, a local breakthrough through the pterygopalatine fossa (arrow) gaining access to the extracranial soft tissue (arrowhead) was observed. Six months after the initial diagnosis (c) and 10 months after the initial diagnosis (d), a new local lymphatic metastasis in the left neck (asterisk) was observed indicating the lymphatic metastization of the tumor. 


\section{Case Reports in Oncology}
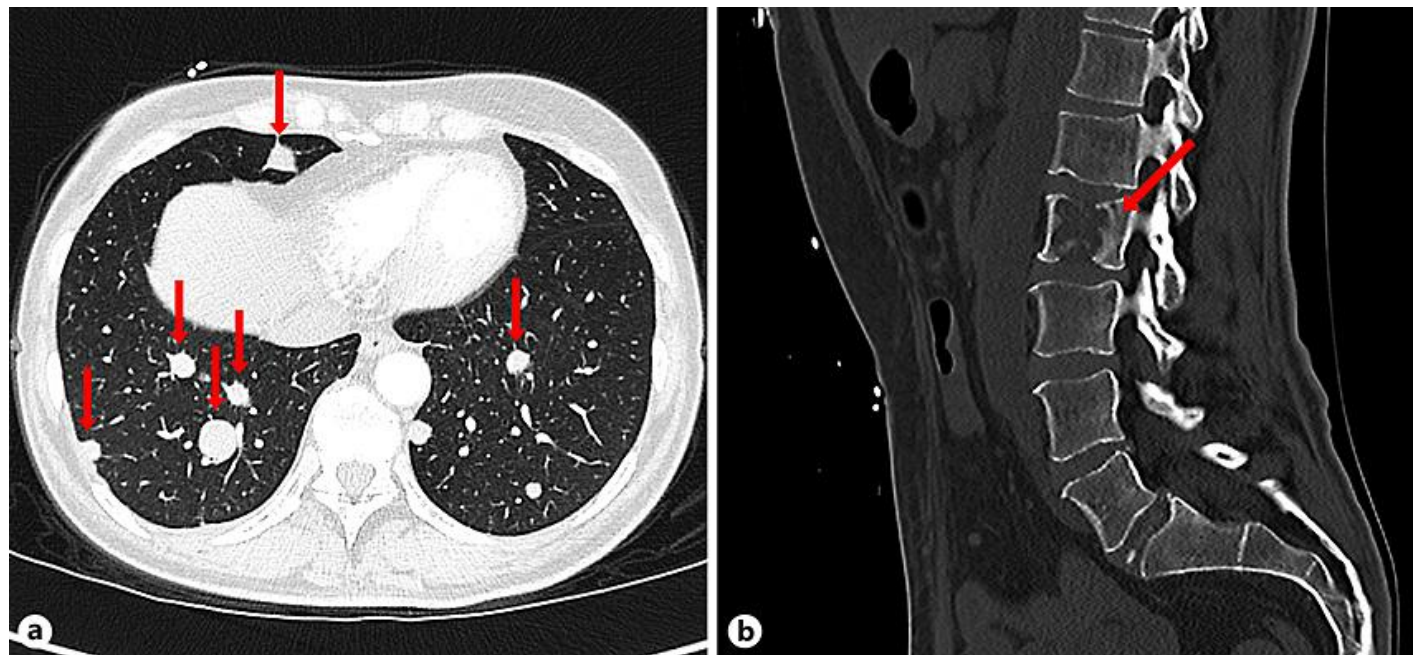

Fig. 2. Extracranial metastases of the GBM. CT shows multiple pulmonary (a) and one osteolytic metastasis located at the second lumbar vertebral body (b).
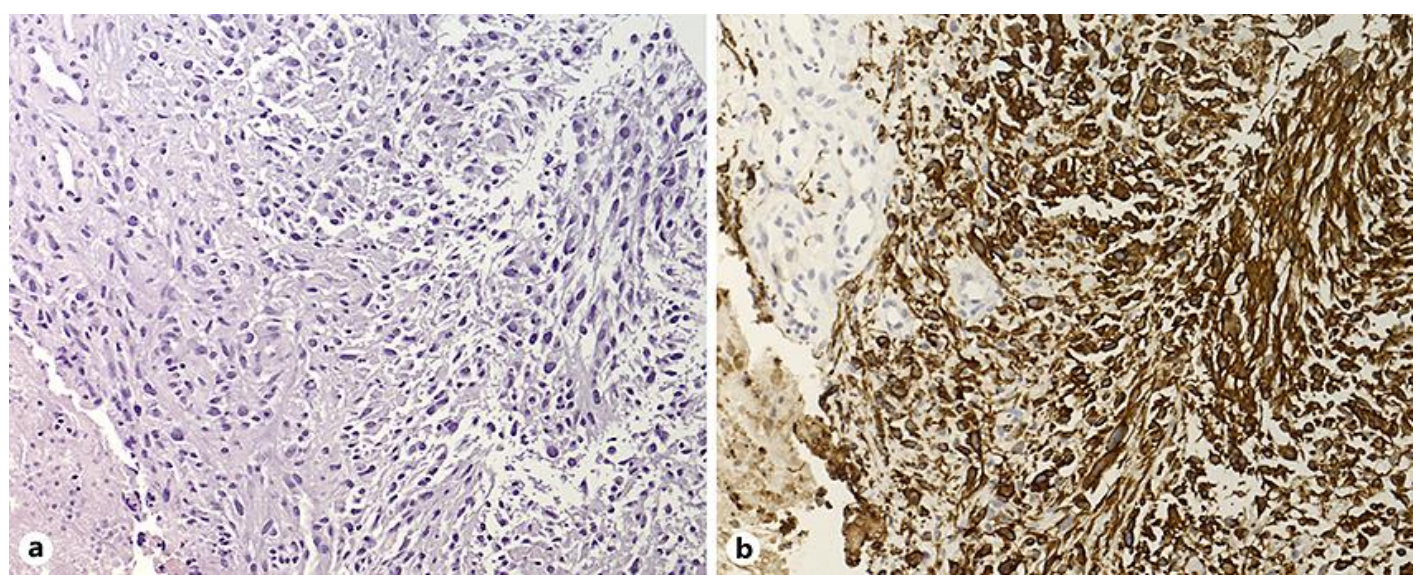

Fig. 3. Histological characteristics of one extracranial GBM metastasis. One lesion located in the subcutaneous tissue below the left costal arch was biopsied for further histological diagnostics. a Light microscopy of a high cellular, pleomorphic, glial tumor with nuclear atypia and focal necrosis. H\&E staining. Original magnification, $\times 200$. b Immunohistochemically, tumor cells show immunoreactivity for the glial fibrillary acid protein. Original magnification, $\times 200$. Morphological and immunohistochemical findings of the metastasis corresponded to those of the intracranial GBM, thus leading to the diagnosis of an extraneural metastasis of the GBM. 
Table 1. Summary of case reports with GBM and 3 or more sites of extraneural metastasization $[1,2,12,13$, $16,17,19,20,33-44]$

\begin{tabular}{lll}
\hline First author [Ref.] & Year & Extraneural location \\
\hline Davis [1] & 1928 & Lung, bone, soft tissue \\
Wisiol [33] & 1962 & Lung, bone, soft tissue \\
Makeever [34] & 1966 & Lung, pleura, diaphragm, lymph nodes \\
Rubinstein [35] & 1967 & Lymph nodes, bone, retroperitoneal, muscle \\
Smith [12] & 1969 & Lymph nodes, bone, liver, pancreas \\
Smith [12] (case 20) & 1969 & Lymph nodes, lung, pleura \\
Wakamatsu [17] & 1971 & Lung, pleura, pericardium, diaphragm \\
Hulbanni [16] & 1976 & Lymph nodes, lung, bone \\
Liwnicz [14] (case 1) & 1979 & Lymph node, lung, pleura, pericardium \\
Liwnicz [14] (case 2) & 1979 & Bone, paravertebral, mesenterium \\
Liwnicz [14] (case 3) & 1979 & Lymph nodes, bone, retroperitoneal, soft tissue \\
Yokoyama [36] & 1985 & Lymph nodes, lung, bone, pleura, liver \\
Horiuchi [19] & 1996 & Bone, orbital/nasal/oral cavity, muscles \\
Wallace [37] & 1996 & Lymph nodes, muscles, eye \\
Beauchesne [38] & 2000 & Lung, bone, heart \\
Houston [39] (case 1) & 2000 & Lymph nodes, bone, mediastinum \\
Houston [39] (case 2) & 2000 & Liver, lung, skin \\
Ueda [40] & 2003 & Lymph nodes, bones, lung, liver, pancreas, kidney, epicardium, soft tissue \\
Mirzayan [41] & 2005 & Lymph nodes, bone, mediastinum, soft tissue \\
Didelot [42] & 2006 & Lymph nodes, lung, bone, spleen \\
Mujic [43] & 2006 & Lung, bowel, pancreas, soft tissue \\
Saad [2] & 2007 & Bone, lung, liver, soft tissue \\
Beauchesne [20] (case 1) & 2011 & Lung, bone, muscle, heart \\
Beauchesne [20] (case 2) & 2011 & Lymph nodes, lung, spleen \\
Beauchesne [20] (case 3) & 2011 & Lymph nodes, lung, liver \\
Hamilton [44] (case 2) & 2014 & Lymph nodes, bone, soft tissue \\
Anghileri [13] & 2016 & Lymph nodes, bone, soft tissue \\
\hline
\end{tabular}

